\title{
Combined Onset of Bilateral Endophthalmitis and Spondylodiscitis as Manifestations of Streptococcus dysgalactiae Infection in an Elderly Woman
}

\author{
Kazuki Takakura, Mikio Kajihara*, Ryoko Fukushima, Seiji Arihiro, Shigeo Koido, \\ Hisao Tajiri \\ Department of Internal Medicine, The Jikei University School of Medicine, Tokyo, Japan \\ Email: ${ }^{*}$ mikio.kajihara@jikei.ac.jp
}

Received 10 January 2014; revised 9 February 2014; accepted 5 March 2014

Copyright (C) 2014 by authors and Scientific Research Publishing Inc.

This work is licensed under the Creative Commons Attribution International License (CC BY). http://creativecommons.org/licenses/by/4.0/

\section{cC) (i) Open Access}

\begin{abstract}
A case of a patient with bilateral endophthalmitis and vertebral infection is presented here. The case presented with fever, general malaise, reduced vision and back pain for at least two weeks. Diagnosis was established by positive blood cultures together with ophthalmology review and radiological findings. Streptococcus dysgalactiae subspecies equisimilis was isolated from blood cultures. This case demonstrates the risks for older patients of developing invasive disease upon exposure to Streptococcus dysgalactiae, even if underlying diseases are absent.
\end{abstract}

\section{Keywords}

Bilateral Endophthalmitis, Spondylodiscitis, Streptococcus dysgalactiae

\section{Introduction}

Endophthalmitis is an uncommon complication of bacterial infections, whilst spondylodiscitis is also a relatively uncommon cause of back pain. Streptococcus dysgalactiae could have aetiological importance in both inflammatory diseases in patients usually with underlying diseases, but such infection could be associated with no primary illness such as diabetes mellitus and heart disease.

We herein describe an extremely rare case of a 78-year-old previously healthy female patient with bilateral

\footnotetext{
*Corresponding author.
} 
endophthalmitis and vertebral infection caused by Streptococcus dysgalactiae.

\section{Case Report}

A previously healthy 78-year-old Japanese woman was referred to us from her physician at a local hospital. She first presented to the local hospital complaining of diarrhoea, abdominal pain, fever and lethargy. She had been under the treatment by her physician for presumptive acute enterocolitis with fosfomycin for a fortnight before she developed eye pain and reduced visual acuity. She also experienced increasing lower back pain. On initial presentation at our hospital, the patient was alert and fully oriented but looked generally unwell, lethargic and pyrexial at $39.0^{\circ} \mathrm{C}$. Her vision was highly deteriorated and limited bilaterally to light and dark perception only. Bilateral conjunctivitis, with erythema and purulent exudate, was present. There was also tenderness over the lumbar vertebrae but no neurological deficit was found on examination.

Initial laboratory investigations revealed white blood cell count of $14.7 \times 10^{9} / \mathrm{L}$ and normal liver and renal functions. C-reactive protein was raised to $228 \mathrm{mg} / \mathrm{L}$. Glucose levels were normal upon repeated measurements with no evidence of diabetes mellitus. The findings from a chest radiograph and urinalysis were normal. CT of the chest and abdomen showed no signs of malignant tumours or active inflammatory diseases, while oesophagogastroduodenoscopy was unremarkable. Although transoesophageal echocardiography was not performed due to deterioration of her general condition, the physical findings and bedside echocardiography showed no particular signs of bacterial endocarditis. We have performed two sets of blood cultures at the time of admission, and both showed gram-positive cocci resembling Streptococcus, later speciation of which revealed a bacteraemia of $\beta$-haemolytic group $\mathrm{G}$ streptococci (Streptococcus dysgalactiae subspecies equisimilis).

Vitreal fluid aspirated from both eyes was purulent, but there was no growth from both direct and enriched cultures. Spinal tap was also performed but cerebrospinal fluid cultures were negative either. Fine needle biopsy of the spine was not performed. Magnetic resonance imaging (MRI) of the head was unremarkable, but spinal MRI, requested to investigate back pain, revealed surrounding inflammatory changes extending into both adjacent vertebral bodies at L4/5, in keeping with infective spondylodiscitis (Figure 1). The ${ }^{67} \mathrm{Ga}$ scan showed areas of increased uptake around both eyeballs and lumbar vertebrae (Figure 2).

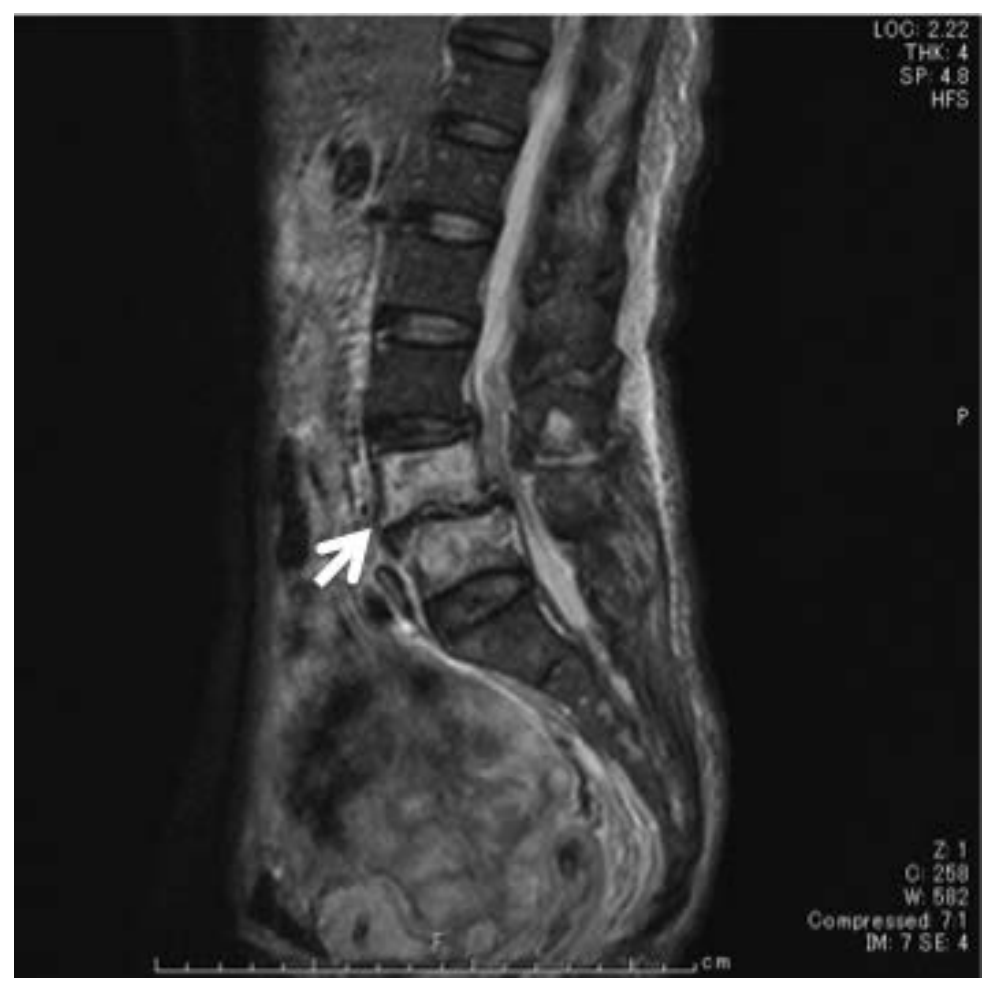

Figiure 1. MRI image showing inflammatory changes at the level of L4/5 (arrow). 


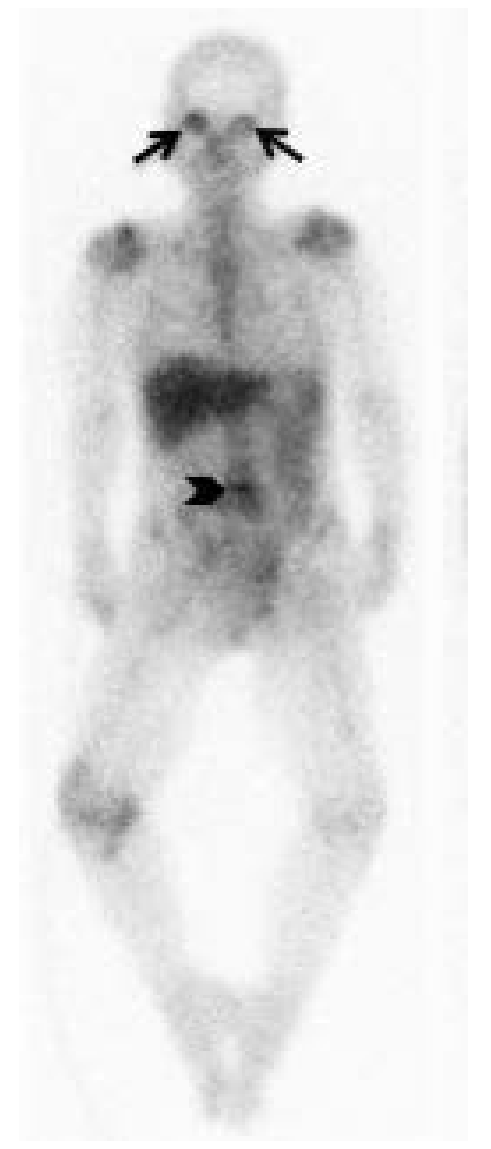

Figure 2. In ${ }^{67} \mathrm{Ga}$ imaging, intense tracer accumulation was observed in the both eyeballs (arrows) and lumbar vertebrae (arrowhead).

The patient was treated aggressively with intravenous fluid hydration and commenced on empiric antibiotics. Initial treatment included intravenous tazobactam/piperacillin for a week, followed by ampicillin and gentamicin for another fortnight, giving a total duration of three weeks of antibiotic therapy. An ophthalmology consult was obtained, and the patient was treated with intravitreal injections in both eyes with ceftazidime. A vitreal fluid aspiration was also performed, but with a complaint of intractable pain in the eyes, the patient eventually underwent a pars plana virectomy.

Outcome was generally favourable. The pyrexia abated, and low-back pain reduced progressively during the course of the medical treatment. The acute inflammatory parameters settled and there was no relapse during the follow-up period. The patient's intraocular inflammation subsided within a few days after vitrectomy, and so did the pain in the eyes as well as headache. The visual acuity, however, did not sufficiently improve and the best correct visual acuity remained counting fingers at $50 \mathrm{~cm}$ in the both eyes when she was discharged.

\section{Discussion}

Streptococcus dysgalactiae can be divided into 5 distinct subtypes, but human disease is most associated with Streptococcus dysgalactiae subspecies equisimilis, Lancefield serogroup G [1]. Empiric antibiotics were started prior to vitreal fluid aspiration and spinal tap, which was thought to have possibly sterilised the aspirates, but blood cultures at admission grew and identified Streptococcus dysgalactiae as the likely pathogen, and septic embolic phenomena to both eyes and vertebrae are considered to have caused the present patient's endophthalmitis and spondylodiscitis.

Endophthalmitis is an uncommon complication of bacterial infections, and although intra vitreal administra- 
tion of antibiotics is recommended, the prognosis for regaining sight in the affected eye remains poor. Bilateral endophthalmitis is even less common, and that as a complication of group $G$ streptococcal endocarditis has been described before in the literature, but only twice to date [2] [3]. Interestingly, in our case, the patient had no known underlying disease, except for preceding abdominal symptoms.

In the meantime, spondylodiscitis is also a relatively uncommon cause of back pain. Infection is believed to arise in most cases by haematogenous spread. Staphylococcus aureus is the most common causative organism but other Gram-positive bacteria could also have aetiological importance in spondylodiscitis patients with underlying diseases such as diabetes mellitus and heart disease. However, rare cases of spondylodiscitis without primary illnes caused by Streptococcus dysgalactiae subspecies equisimilis have also been reported [4], which correspond with the present case.

To the best of our knowledge, this is the first report of combined onset of bilateral endophthalmitis and spondylodiscitis as manifestations of Streptococcus dysgalactiae infection subsequent to acute enterocolitis in a patient with no underlying diseases. The current case demonstrates the risks especially for older patients of developing invasive disease upon exposure to Streptococcus dysgalactiae subspecies equisimilis, even if classical underlying diseases such as diabetes mellitus or actual known cardiac diseases are absent.

\section{Conflict of Interest}

The authors state that they have no conflict of interest (COI).

\section{References}

[1] Vandamme, P., Pot, B., Falsen, E., Kersters, K. and Devriese, L.A. (1996) Taxonomic Study of Lancefield Streptococcal Groups C, G, and L (Streptococcus dysgalactiae) and Proposal of S. dysgalactiae subsp. Equisimilis subsp. nov. International Journal of Systematic and Evolutionary Microbiology, 46, 774-781. http://dx.doi.org/10.1099/00207713-46-3-774

[2] Gopalamurugan, A.B., Wheatcroft, S., Hunter, P. and Thomas, M.R. (2005) Bilateral Endophthalmitis and ARDS Complicating Group G Streptococcal Endocarditis. Lancet, 366, 2062. http://dx.doi.org/10.1016/S0140-6736(05)67817-8

[3] Wickramasinghem, N. and Harris, K. (2010) Bilateral Endophthalmitis as a Primary Manifestation of Streptococcus dysgalactiae Endocarditis and the Role of 16S rDNA Polymerase Chain Reaction in Identification. Diagnostic Microbiology \& Infectious Disease, 67, 185-187. http://dx.doi.org/10.1016/j.diagmicrobio.2010.01.008

[4] Kumar, A., Sandoe, S. and Kumar, N. (2005) Three Cases of Vertebral Osteomyelitis Caused by Streptococcus dysgalactiae subsp. Equisimilis. Journal of Medical Microbiology, 54, 1103-1105. http://dx.doi.org/10.1099/jmm.0.46061-0 